\title{
Managing the opioid epidemic: back to the basics with resuscitation
}

\author{
Orlando Hung*; Ronald D. Stewart ${ }^{\dagger}$; Gemma Malpas*; Sarah Phipps*; David Hung ${ }^{\dagger}$
}

\section{INTRODUCTION}

Opioid-related overdoses claimed the lives of almost 4,000 people in Canada in 2017. ${ }^{1}$ Despite bystander naloxone administration and overdose education programs, which became available in Canada in $2016,{ }^{2}$ the death toll continues to climb. Faced with the alarming number of opioid-related deaths, we must make it our priority to reduce the risk of death for opioid users. A more effective approach would require modifications to our current practices to complement and strengthen programs emphasizing the early use of naloxone.

Intramuscular or intranasal administration of naloxone can reverse the effects of opioids. However, it may be of no benefit to an unresponsive patient with no detectable respirations or cardiac output. We assert that the priority for managing a patient who is unresponsive because of a suspected overdose is first to establish immediate oxygenation and circulation (i.e., airway, breathing, and circulation $[\mathrm{ABC}])$. While most free "naloxone packages" clearly direct the rescuer to call for help and to support the ABCs before administering naloxone, many lay rescuers do not understand the importance of first calling for help and performing cardiopulmonary resuscitation (CPR). The emphasis on the administration of naloxone without first urging the performance of CPR may cost the lives of those in cardiac arrest and patients who may be unresponsive because of a condition other than that of an opioid overdose.

Using healthy apneic volunteers, Safar et al. demonstrated conclusively that sufficient ventilation could be achieved by laypersons, using the mouth-tomouth technique. ${ }^{3}$ Citing these findings, Safar et al. were instrumental in developing CPR that was quickly validated and implemented worldwide. The widespread public adoption of CPR has been fundamental to improving the outcomes of out-of-hospital CPR and has saved countless lives. ${ }^{4}$

Despite this life-saving procedure, recent studies have reported the reluctance of bystanders and health care providers to perform mouth-to-mouth ventilation (MTMV), ${ }^{5}$ even though the number of infections related to resuscitation is estimated to be less than 1 in $200,000 .{ }^{6}$ Recognizing the reluctance of the public, the American Heart Association (AHA) now recommends that untrained rescuers change the sequence from the traditional $\mathrm{ABCs}$ to chest compressions, followed by airway and breathing $(\mathrm{CAB}){ }^{7}$ This change is designed to encourage more rescuers to begin $\mathrm{CPR}$ using a compression-only technique, particularly if only one rescuer is available. Data from the nationwide dissemination in Japan comprising recommendations for continuous chest compression CPR for lay rescuers showed that the implementation of compression-only CPR improved bystander CPR rates and increased survival. $^{8}$

Regardless of a rescuer's level of training, chest compressions should be urgently performed on any unresponsive and pulseless patient, after ensuring the rescuer's personal safety and calling for help. If ventilation and circulation are present in the unresponsive patient, the rescuer should next ensure that the airway is patent using a chin lift and jaw thrust and, then, simply turning the patient on the side. After ensuring adequate ventilation and circulation, the rescuer can then begin to determine the probable cause of unconsciousness and administer naloxone, if indicated.

From the *Department of Anesthesiology, Dalhousie University, Halifax, NS and tDepartment of Emergency Medicine, Dalhousie University, Halifax, NS.

Correspondence to: Dr. Orlando Hung; Department of Anesthesia, Dalhousie University, Queen Elizabeth II Health Sciences, 1278 South Park St, Halifax, NS, B3H 2Y9; Email: hung192@gmail.com

(C) Canadian Association of Emergency Physicians 
It is understandable that many rescuers may be reluctant to call for help in a situation in which it could be assumed that both the rescuer and the patient would be subject to scrutiny by law enforcement agencies. To address this frequently expressed concern, the Parliament of Canada recently (2017) passed legislation, "The Good Samaritan Drug Overdose Act," that protects patients, rescuers, and anyone calling 911 for a suspected drug overdose from being charged with drug possession. We would suggest that this fact be prominently displayed in large print on naloxone kits and posted prominently on websites and in appropriate public spaces.

Our approach to this opioid epidemic for this vulnerable group of patients begins with, and should be accompanied by, a widespread public revitalization of citizen CPR programs. To engage public support, the AHA recently recommended implementing the "Kids Save Lives" program to educate schoolchildren on CPR, calling it a "Civic Duty." ${ }^{10}$ Several US states have begun to teach schoolchildren CPR. Recently, medical students at Dalhousie University in Halifax initiated a new program called PUSH that teaches the basics of the compression-only CPR course to schools and organizations within the community. Their initiative focuses on accessibility and outreach but does not provide official CPR certification (www.pushforlife. org). In addition, social media can also be used to engage the public to teach them how CPR impacts the naloxone program. Recently, the Resuscitate App on Android operating system phones offers instant videos on how to perform CPR, use an automated external defibrillator (AED), aid a choking victim, and save lives. The renewed focus on CPR should form the basis of awareness programs in opioid dependence clinics and other areas specific to these high-risk groups.

In summary, we believe that launching a naloxone program to address the resuscitation of patients with suspected opioid overdoses must be accompanied by a renewed educational program on CPR for the unconscious patient, in addition to training on safety and the proper use of naloxone. That is, the approach to an unresponsive patient for whom the cause is unknown should include performing an immediate scene safety assessment for the patient and rescuer, calling 911, and, then, employing CPR based on the rescuer's level of training or along with instruction from 911 dispatch. If an opioid overdose is suspected, naloxone can be administered provided that adherence to CPR guidelines are followed first. The success of a renewed and widespread citizen CPR training program requires the support of our communities. With proper education and communication, this approach, combined with a well-planned naloxone program, will save more lives.

Acknowledgements: OH, RS, GM, SP, and DH prepared the manuscript.

Keywords: cardiopulmonary resuscitation, CPR, naloxone, opioids, overdose, resuscitation

Competing interests: None.

\section{REFERENCES}

1. Special Advisory Committee on the Epidemic of Opioid Overdoses. National report: Apparent opioid-related deaths in Canada. Ottawa: Government of Canada; 2018.

2. Access to naloxone in Canada (including NARCANTM Nasal Spray). In: Products (Da H, ed). Ottawa: Health Canada; 2016.

3. Safar P, McMahon M. Mouth-to-airway emergency artificial respiration. 7 Am Med Assoc 1958;166(12):1459-60.

4. Gu XM, Li ZH, He ZJ, Zhao ZW, Liu SQ. A meta-analysis of the success rates of heartbeat restoration within the platinum $10 \mathrm{~min}$ among outpatients suffering from sudden cardiac arrest in China. Mil Med Res 2016;3(1):6.

5. Wenzel V, Lindner KH, Prengel AW. [Ventilation during cardiopulmonary resuscitation (CPR). A literature study and analysis of ventilation strategies]. Anaesthesist 1997;46 (2):133-41.

6. Mejicano GC, Maki DG. Infections acquired during cardiopulmonary resuscitation: estimating the risk and defining strategies for prevention. Ann Intern Med 1998;129 (10):813-28.

7. American Heart Association. American Heart Association Focused Updates on Adult and Pediatric Basic Life Support and Cardiopulmonary Resuscitation Quality; 2017.

8. Iwami T, Kitamura T, Kiyohara K, Kawamura T. Dissemination of chest compression-only cardiopulmonary resuscitation and survival after out-of-hospital cardiac arrest. Circulation 2015;132(5):415-22.

9. Good Samaritan Drug Overdose Act. In: Canadian Department of Justice, ed. Ottawa; 2017.

10. Böttiger BW, Semeraro F, Wingen S. "Kids Save Lives": educating schoolchildren in cardiopulmonary resuscitation is a civic duty that needs support for implementation. $7 \mathrm{Am}$ Heart Assoc 2017;6(3):e005738. 